\title{
Research and Practice in Developing Historical Materials in Teaching Calculus
}

\author{
Yi-Wen Su
}

\begin{abstract}
In this study, we plan to jointly develop historical materials of calculus by educators and inservice teachers, hoping this will facilitate its use by field teachers, and through this approach, students can better understand the concepts and development of mathematical thinking, as well as foster the students' interest in learning and conceptual understanding. The study of history of calculus is mainly divided into three: "Connotation of Limit," "Connotation of Derivative," and "Segment and Integral. From the research process and results, it has been found that the developed history of calculus materials have a positive effect on the students, which also shows the feasibility of integrating the history of calculus material into the high school mathematics teaching.
\end{abstract}

Index Terms - Calculus, limit, history of mathematics.

\section{INTRODUCTION}

This research originated from a study conducted by the Ministry of Science and Technology (MOST), the first of which is "The practice and research of integrating math reading into teaching". The present project is aimed at developing an in-depth study into senior high school students' math reading curriculum. In the process of the research we realized that learning math became more interesting when we integrate historical readings to its content, with students discussing and clarifying mathematical concepts. The other research project of the MOST was "The Strategies and Teaching Practices of Reading Comprehension of Geometry Text." The study developed the following topics in Geometry: "The transversal line properties of parallel lines." With practical applications in the teaching, we discovered that Junior high school students were better able to understand the math topics when math reading materials were integrated into the lesson. The third project was "Developing and practicing elementary math cultural material." This study was conducted among 77 first grade elementary students for one year, helping them to understand mathematical symbols, which earned the support and appreciation of both parents and pupils.

The author began teaching in the university in 2007 , acting primarily as adviser to graduate students who were potential primary school teachers; thus, it was then that the author's interest and attention towards math reading materials development and research arose. In integrating history of mathematics in the curriculum for elementary, junior and

Manuscript received March 5, 2018; revised September 23, 2018. This work was supported by the Ministry of Science and Technology of Taiwan, under grant number MOST 105-2511-S-845-008-MY3.

Yi-Wen Su is with the Department of Mathematics, University of Taipei, Taiwan (e-mail: yiwen@utaipei.edu.tw). senior high schools, we have discovered that the mystery and secret of math were uncovered by the students. Not only were the students able to understand the origins of math concepts, it also improved and reinforced the students' attitude toward math learning [1]-[3]. From the above mentioned experience, the author learned to appreciate how integration of historical materials in actual teaching can enhance students' understanding of mathematical concepts when they are linked to social and cultural contexts in a variety of human activities, whether the learners are high school students or even elementary pupils [4], [5].

The mathematics curriculum guidelines in the United States over the recent years have always attached great importance to the humanistic aspect of mathematical knowledge. For instance, as early as 1989, the National Council of Teachers of Mathematics (NCTM) already pointed out the value of helping students realize the interaction between mathematics and its historical context, its impact on culture and life [6]. Again, in the year 2000, mathematics is one of the greatest cultural and intellectual achievements of mankind in this fast-changing world. Citizens should develop an appreciation and understanding of this achievement, including their beauty and even tts entertaining aspects [7]. Fasanelli (2000) [8] analyzed the curricula of China, Greece, Italy, the Netherlands, Poland, Australia, Brazil, Denmark, France, New Zealand, Norway and other countries and found that the history of mathematics can inspire one's affection, cognition and culture activities. Lange (2003) [9] considered mathematics a top priority from a historical, philosophical and social point of view. Niss and Højgaard (2011) [10] also argued that mathematics cannot be viewed as a theoretical discipline, but should also emphasize links with other fields, especially with culture and society, especially the history of mathematics and statistics.

From the above description, we can see the importance of mathematical literature in the field of math teaching. However, as far as actual practice is concerned, it is not easy to search for related materials in the history of mathematics. Even if teachers are determined to implement it, the first challenge they face is where to look for the material [11]. An online survey of 367 secondary school math teachers by Panasuk and Horton (2012) [12] also showed that while most teachers agree with the value and place of history of mathematics in math learning, the lack of resources related to history of mathematics is one of the main reasons why history of mathematics in the classroom is hardly used.

Thus, in this study, we plan to jointly develop historical materials of calculus by educators and inservice teachers, hoping this will facilitate its use by field teachers, and through this approach, students can better understand the concepts and development of mathematical thinking, as well 
as foster the students' interest in learning and conceptual understanding. The research topics of this study are as follows:

1) How can we develop historical materials of calculus?

2) What is the teaching practice of the historical materials?

\section{REVIEW OF RELATED LITERATURE}

In the following pages, we shall discuss the theories and strategies of how the history of mathematics have been integrated into mathematics teaching, as well as the connection between mathematics and narrative, which can be divided into the following: history of mathematics and teaching mathematics; mathematics and its relation to narrative.

\section{A. Study of History of Mathematics and Mathematics Teaching}

Since the 1970s, mathematics education community has begun to pay attention to the trend of integrating history of mathematics in mathematics teaching. The International Study Group on the Relations between the History and Pedagogy of Mathematics (HPM) of the International Commission on Mathematics Instruction (ICMI) meets every four years to study and promote the connection between history of mathematics and mathematics teaching. That is to say, HPM is an application of mathematical historiography to mathematics education. Its aim is to make use of the research achievements of history of mathematics and the interaction between history of mathematics and mathematics education so as to improve the teaching quality of mathematics teachers and the learning outcomes of students. In recent years, NCTM of the US has encouraged teachers to use history of mathematics activities into math teaching or related courses in history of mathematics. They also provide funding for inservice teachers who sponsor and share experience in history of mathematics courses (http://www.nctm.org/Grants-and-Awards/grants/

Professional-Development-Scholarship-Emphasizing-the-Hi story-of-Mathematics/), and Taiwan has issued an "HPM Newsletter" $\quad$ since 1988 (http: http://math.ntnu.edu.tw/ horng/letter/hpmletter.htm) in line with the international academic community, paying close attention to the integration of the history of mathematics into the subject of mathematics teaching.

There are many reasons for incorporating historical dimensions into math teaching, such as stimulating students' interest, giving them a better understanding of concepts and theories, multiculturalism and aiding them with math learning [13]-[17]. In sum, the goal of integrating history of mathematics into mathematics teaching can cover three categories: affective, cognitive and cultural activities. Affective aspects include inducing learning motivation, arousing interest in learning, and inspiring the growth of students' personality through the biography of mathematicians. In the cognitive part, it helps students to learn through historical context the development of mathematical concepts, allowing students to compare different problem solving methods or ways of thinking, and liberating them from a single approach of mathematical thinking. As for the cultural activities, we can achieve meaningful learning by recognizing the development of mathematics with distinctive characteristics from all ethnic groups, appreciating and cherishing the varied cultural characteristics of mathematics [8]. Historical material references in mathematics can include affective, cognitive and cultural aspects. Providing teachers with suitable historical clips and texts with teaching examples that help students understand and inspire them with important concepts of mathematics could serve to introduce them appropriately into mathematics teaching [11].

For example, the use of unit fraction is a very important mathematical feature in the development of mathematics in ancient Egyptian culture. Streefland (1991) [18], for example, cited the topic "Divide eight loaves among ten men" from Rhind Mathematical Papyrus of Ancient Egypt, as follows unit fraction $\frac{8}{10}=\frac{1}{2}+\frac{1}{5}+\frac{1}{10}$ give students an idea of arithmetic of fractions in Egyptian math: all fractions are expressed in units, which is a fairly good example of how mathematics is evolving from everyday life. Using the original texts, one can use only illustrations or images to implement, getting the students to narrate or describe them to help understand the mathematics behind [19], or Pengelley's (2011) [20] example of mathematical induction and combinations of Pascal, where students not only acquired mathematical knowledge, but they also appreciated the convenience of modern symbols.

The materials of the history of mathematics developed in this project mainly focus on the historical context of the development of mathematical thinking as well as the mathematical knowledge produced by various ethnic cultures. In the classroom, teachers can share the concern towards mathematics culture with students as described by some researchers [17], [21]. The following are examples with detailed description:

1) Biographies of mathematicians and their struggles can be discussed in class. For example: $\mathrm{Zu}$ Chongzhi and his son tried very much to divide a circle until finally they proposed the approximate value of pi (П) 355/113, which led to some outstanding global accomplishments, inspiring students' interest in learning from the masters, taking pride in their goodness.

2) A fitting introduction to the origin of mathematical symbols or mathematical concepts to help students realize the relationship between mathematical knowledge and human activities. That is to say, by tracing and exploring the memory of these ancient scholars, which combined creativity and culture, students will be able to understand the path of their mathematical thinking, arousing their interest and inspiration. For instance, one can trace the thought process from the history and development of imaginary numbers, from Cardano (1501-1676) and Bombelli (1526-1572), to the solution of the cubic equation, where they had to face complex numbers for the first time, taking mathematicians centuries to accept this idea in the 19 th century.

3) We should make good use of ancient texts to enlighten students, enabling them to reflect on the past as well as the present, finding similarities and differences between 
the present strategies and their originals. In other words, we can find appropriate historical materials related to the textbook unit that shows the development of some mathematical concepts as a reference for students. For example, the area of a triangle $\frac{1}{2} a b \sin C$ in the high school curriculum, then, $\sin \mathrm{C}=\sqrt{1-\cos ^{2} C}$ which can be substituted with the $\cos \mathrm{C}=\frac{a^{2}+b^{2}-c^{2}}{2 a b}$ to find the area of a triangle $\Delta=\sqrt{s(s-a)(s-b)(s-c)}(a, b, c$ are the three sides of the triangle, and s stands for half of the perimeter). Teachers can then supplement the original Heron's proof, which is one of the most beautiful mathematical proofs in elementary geometry.

4) Using texts to give students a variety of methods to solve problems or different approaches to the problem, debunking the singular approach to math. For example, there are many proofs of the Pythagorean Theorem, from Euclid's idea of The Elements of congruent triangles to China's Zhao Shuang's method of direct calculation the area of the diagram.

5) Using appropriate texts conflicts can arise in students' cognitive understanding, and thus needs to be clarified; for example, in teaching probability using the problem of points by mathematicians Luca Pacioli (1445-1517), Filippo Calandri (1467-1512), Pierre de Fermat (1601-1665), and Blaise Pascal (1623-1662), students will be given the opportunity to discuss and explore.

6) Searching for materials that can be linked to other fields of study can enrich math learning. For instance, arranging math poem materials, from analyzing ancient math poems to appreciation of modern math poems, and then allowing students to compose their own math poems; this can be connected with literature and thus achieve the goal of integrating various fields of study.

7) By recognizing the distinctive mathematical development presented by various ethnic groups, we can cherish the cultural roots of mathematics. For example, by introducing how local mathematicians in Japan developed and promoted their math by Sangaku, forming a unique aspect of Japanese mathematics so that students can understand different regions will produce different math styles.

Dutch mathematician Freudenthal (1973) [22] consider mathematics as a human activity, which must be connected to reality, close to children's experience and socially relevant. Gravemeijer (1989) [23] proposed three heuristic teaching designs, guided reinvention, didactical phenomenology, and emergent modeling. The principle of reinvention first suggested that instructional designers focus on the history of mathematics to understand the exact development of mathematics over time, and to suggest that it is necessary to explore whether it is possible to anticipate more formal math from the informal explanation and solution of the student. If the answer is yes, then the student's initial informal reasoning can serve as a starting point for the reinvention process. Using historical resources, teachers can effectively provide and support students' learning in different situations [24]. In fact, many links in different parts of mathematics do really exist, and paying attention to history is one way to perceive these links. For us and students, knowing the history of a concept can lead to a deeper understanding. Active participation of students can improve their sense of accomplishment in mathematics, which evidently shows the importance of the history of mathematics. So how can we use history of mathematics and rewrite abstract cold mathematical symbols and concepts to attract people to read it? In the following pages, the researcher will elaborate on the connection between math and narrative.

\section{B. Mathematics and Narrative}

History and story are clearly related, and the earliest forms of passing on past evidence were storytelling [25]. Storytelling is the oldest form of communication and the story is a combination of messages and events that capture the soul. Sometimes, the storyteller must search through books, ideas, and writings to find the perfect material. Only through mining, sorting and processing can we transform the material into the beginning of a story. Corfield (2012) [26] describes narratives as a series of structured designs in sequence, not necessarily the traditional storytelling, characters, settings, and so on. The difference between scientific thinking and narrative thinking lies in that scientific thinking leads to an external description of the original context, while narrative thinking preserves the contextual richness. The difficulties in doing math are mainly the gaps in narrative, the lack of stories [19]. In recent years, some scholars have noticed the relationship between mathematics and narrative [27], [28]. They think solving problems or reasoning can be compared to narration in novels, that there is a very important link between mathematical thinking and stories, and they also hope to show the story of mathematical thinking development. Doxiadis (2005) [29] examined the similarity between narrative and proof, contrasting the construction of narratives with the construction of proofs and found that both can be a metaphor for a journey. Good mathematical concepts that can be used to prove that some arguments, a good story, have different ways of enlightening people [30]. If we can link the two, we should be able to promote the popularization of mathematics.

From the above, we can see that in mathematics learning, the combination of mathematics and narrative is a topic of special concern, and some mathematicians are also actively linking the two. For example, Professor John Allen Paulos, a professor of mathematics and a best-selling author, explores sparks of encounters between mathematics and stories on his ABCNEWS.com monthly column at ABC News (http://abcnews.go.com/Technology/WhosCounting/story?id $=711316$ \& page $=3$ ). In the European region, several seminars on mathematics and narrative have been organized, allowing participants to freely discuss many of the possibilities of mathematics and story. In 2012, they collected works by mathematicians, historians, philosophers and writers attending conferences and published a book entitled, "Circles Disturbed: The Interplay of Mathematics and Narrative," which explored the connection between mathematics and narrative. In addition, Alex Kasman, a professor of mathematics, has also set up a mathematical novel website 
(http://kasmana.people.cofc.edu/MATHFICT/). The site collects more than a thousand mathematical short stories, plays, novels, films and comic books. Its homepage reads: "Do you like fiction and mathematics? Are you looking for a book or story that might be useful for the students in your math class? Are you interested in what our society thinks about mathematicians? Then you've come to the right place." One can see that efforts to narrow the distance between mathematics and novels is on the rise.

The combination of mathematics and narratives arouses the interest of primary and secondary school teachers and students and the general public in mathematics, reducing their sense of fear and it is hoped that they appreciate the beauty and order of literary narrative [31]. In this project, we focus on how to develop history of mathematics materials to aid mathematics learning through the proper combination of mathematics and narrative.

\section{MeTHODOLOGY}

This project is a qualitative study where the teacher educator and secondary school teachers worked and discussed together the development of teaching materials on the history of calculus. In this study, the research team discussed the pursuit of relevant historical materials of calculus, compiling them, devising the lesson plan, then implementing them in the classroom, and finally, observing the students' reactions. The researcher was a participant, a consultant, and an observer. Besides attending the faculty meetings, the researcher, acting as consultant, discussed with the participating teachers' questions or difficulties they encountered in the classroom.

The method of data collection in this study is mainly through "collection of texts". The text contains the minutes of the meeting, which was held every two weeks, the feedback sheets from the students, and the experiences and notes of the participating teachers. From these data, we could know more clearly the suitability of the history of calculus materials that had been developed.

The research work was divided into three phases: "Textual Study," "Guidelines for Developing Lesson Plans," and "Evaluation of Teaching Effectiveness." Below is the detailed explanation.

\section{A. Textual Study}

The author had taught in high school before and had tried many times to incorporate history of mathematics into math teaching. She currently teaches history of mathematics and math education majors in the mathematics department of a university and also teaches general education courses in its general education center. From this she has accumulated some materials and experience in designing history of mathematics texts. At this stage, the researcher and participating teachers conduct textual analysis. Each text analysis provided an in-depth analysis rather than an extensive one, and the main tendency was to focus on a detailed analysis of single text. Because textual analysis includes careful attention to detail and rigorous questioning, the reader needs to be guided not to make immediate judgments and should be encouraged to come forward with supporting evidence of their point of view. This type of analysis goes from "familiarity to strangers" since these mathematical concepts were originally familiar to teachers. The research team here had to look carefully at how these concepts were laid out and wondered why they were assembled in this way, exploring how to think about the texts as being "read." All materials will be considered for the different stages of learning ability and literacy indicators, and how they can be properly converted into a lesson plan for teachers.

\section{B. Guidelines for Developing Lesson Plans}

This part refers to the guidelines disclosed by the Center for Research on Teaching and Learning (http://www.crlt.umich.edu/gsis/p2_5). Before starting the lesson plan, one must consider the following three key components: Objectives for student learning; Teaching/learning activities; and Strategies to check students' understanding. As for the lesson plans, the first is to list the "Outline learning objectives;" then "Develop the introduction;" followed by "Plan the specific learning activities," keeping in mind the students' levels of understanding; next is the "Plan to check for understanding;" and finally "Develop a conclusion and a preview." The lesson plans in this study follow these aforementioned guidelines.

\section{Evaluation of Teaching Effectiveness}

After the lesson plans have been tested for validity, the participating teachers will try to use them in the classroom. Then, evaluation can follow using students' worksheets and the teachers' reflections.

\section{RESUlTS AND DisCUSSIONS}

The study of history of calculus is mainly divided into three: "Connotation of Limit," "Connotation of Derivative," and "Segment and Integral," which will be described as follows:

Connotation of Limit: The main objective of this lesson plan is 1) to understand the concept of infinity, and 2) to understand the meaning and purpose of the limits. Since this is the first contact of the students with Calculus, they must necessarily face the concept and significance of limits. The lesson plans to make use of Zeno's paradox so that students think about after an object or curve that has been continuously divided, what is it that remains? How does one express it? What's the problem? Thus, it introduces the significance and necessity of limit and establishes a motivation to learn limits.

Regarding the lesson plan for "Connotation of Limit," instructors implement the lesson and worksheet in line with the progress of the curriculum when they enter last year of senior high school, in which they given a question and answer handout. Because of the time and schedule factors, the teachers may use instant question and answer method by asking for students to volunteer or ask directly anyone to answer. Therefore, after they have answered the questions one writes down their answers on the board. In response to 
the question: "Do you think that when we divide an object, can we continue the endless subdivision, or we can only divide it into its last smallest indivisible components? Why?" Students generally agree that the object can be infinitely divided. Such knowledge should be helpful in learning the limits because no whether in series, progression, or function, an infinite process is involved, whether it endlessly grows bigger or smaller. This endless "process" in understanding limits should not pose any difficulty.

On the second question "For each of these two paradoxes, do you think it reasonable? Please write your reason for agreeing." With regard to the paradox of Zeno, students generally answer that speed and time be taken into account, because time is continuous and thus oppose the arrow paradox. According to the students' answers, compared with the continuity of the function, students will understand why the continuous function needs to be considered in calculus. In teaching this lesson plan, we can link this question to the necessity of continuous functions.

On the third question: "Do you think $0 . \overline{9}=0.9999$ is less than 1 or equal to 1 ? Please write down your reasons." When the students have not been taught the limit of series or progression, they will generally think $0 . \overline{9}<1$ no matter how many digits follow $0.99999 \ldots<1$. Therefore, the teacher reflected over her teaching on the need to further explain the understanding of the definition of actual infinity and the concept of limit to let students plainly realize why there is the so-called sufficiently close meaning in mathematics.

On the lesson of "Connotation of Limit," the research team implemented the teaching practice in another school. The students were two senior high school class sections majoring in science, with 41 students and 37 students in each class. When the teacher did the lesson the students still had not learned the concept of limits.

As mentioned above, since the lesson plan of "Connotation of Limit" was designed hoping to enable students to understand that people have many myths regarding infinity it was necessary and very important to discuss the concept of limit. The learning unit had two problems: a) Zeno's paradox; b) the potential infinite and the actual infinite. By discussing these problems students were able to explore the difficulties one encounters in grasping the concept of infinity. During the teaching process, the teacher adopted the first version of the learning unit because it was not easy for students to comprehend the description of Zeno's Paradox. So, the teacher spent some time clarifying the doubts of the students (especially the Arrow Paradox), and asked the students to answer the problem according to their own understanding. When examining their classmates' answers, one discovered that the knowledge of physics affected their perception of the problem.

When explaining potential infinity and actual infinity, the teachers found out that there was not much effect on the problem whether " $0 . \overline{9}=0.9999 \ldots$ is less than 1 or equal to 1?" When students were discussing the rationality of the two algorithms, there was no doubt regarding potential infinity or actual infinity. Similarly, teachers asked the students to answer according to their own understanding whether the algorithm was reasonable.
As regards "Connotation of Derivative," the main goal of this lesson plan was to 1) understand the relationship between the secant and the tangent, and 2) to understand the origin of the definition of the derivative. The main idea was to use Newton's method to understand why you need to define the derivative in the following formula $\lim _{x \rightarrow a} \frac{f(x)-f(a)}{x-a}$ and the geometric meaning that the derivative represents in solving the tangent problem and to understand the meaning of the derivative from the context of its historical development.

During the teaching of "Connotation of Derivative", teachers work in accordance with the curriculum before the students learn the definition of derivatives. The original lesson plans were designed using Fermat's and Newton's methods in the same worksheet. Students were made to understand the definition of derivatives through the necessity and the implications of small increments using Fermat's and Newton's methods. After the implementation of the lesson, the teachers found out that more time was needed to tackle the subject and the amount of the content also exceeded the students' capacity. Therefore, after the teaching and after discussion, it was decided that some details of the lesson plan be modified into two parts. Newton's method is used to explain the meaning of the derivative. Then, we used Fermat's method of determining maxima and minima to explain why the derivative of the extreme point is zero.

Implementing instant $\mathrm{Q} \& \mathrm{~A}$, the teachers explained the method, and once the students understood it, they wrote down the answers to the questions. The teachers thought this method of teaching had several advantages: 1) it saved time when implementing the lesson plan and study schedule; 2) it allowed the teachers to interpret the contents of the learning unit correctly and made it fully understandable; 3) the students wrote down the answers to questions according to their level of comprehension when the course modules were conducted, which were properly controlled; thus, strengthening and supplementing their learning. From the class response and content, one can see that almost all could reach the goal of learning from a single study unit. After the end of the learning unit and after the teaching, one can properly link the learning content to match the explanation.

The main objectives of the lesson on "Segment and Integral" are the following: 1) to understand the need of using segment in computing for area; 2) to understand the concept of segment; 3) to understand the relationship between segment and integral; and 4) to know Leibnitz's argument in understanding the fundamental theorem of calculus. At the beginning of the development of calculus, the indivisibles and infinitesimals were introduced in handling the calculation of extreme values, tangents, areas, volumes, and so on. This lesson plan allows students to gain an understanding of the strengths and weaknesses of the indivisibles method from Cavalieri, which in turn enables students to understand the need to divide the area into smaller parts for volume and area, and to use Leibnitz's symbolic meanings and interpretation of integral. This allowed students to understand the practice of integral and the significance of the material from a simple history of mathematics.

The lesson on "Segment and Integral," follows closely the 
course syllabus and conducts the lesson plan according to the worksheet before delving into its concepts. This lesson was originally designed to use a few examples with the goal of allowing students to understand the use of segment and approximation in obtaining the area under the curve. Therefore, Cavalieri's principle was explained first, and then they became more familiar with the circular area as an example. The major problem of the segment was discussed; that is, how the process and result of the approximation is used, and finally the example of Leibnitz's method to illustrate the concept of integral and the meaning of the symbols. After the class implementation, it was found out that the original design content took a considerable amount of class time; that is, class period in high school is not enough. So, after discussion, we decided to design a shorter version, using only Leibnitz's integral, explaining with emphasis the understanding of the integral symbol and the fundamental theorem of calculus.

In "Leibnitz's Segment and Integral" lesson plan, the main teaching objectives were: 1) to understand the need of using segment to find the area; 2) to understand the concept of segment; 3) to understand the relationship between segment and integral; and 4) using Leibnitz's method to understand the fundamental theorem of calculus. The lesson plan is mainly designed to explain the beginning of the development of calculus, which arises from indivisibles to infinitesimals when dealing with the extreme values, tangents, areas, volumes, and so on. The study unit began with finding the area of a circle, which introduced the students to understand the necessity of dividing the area into the smallest parts when solving for its area or volume. From this simple practice, students could learn the significance of understanding integral by using Leibnitz's symbols and the explanation of the integral from the history of mathematics materials.

Similarly, using Q\&A and after the teacher's explanation of the method, students wrote down the answer according to their level of understanding. Some of them understood the classical literature, while other students could make out the meaning on their own. When teaching the learning unit on integral, one could easily match the students' understanding of the lesson. After asking some questions, one could sense that this method had helped the students in understanding the fundamental theorem of calculus.

\section{CONCLUSION}

In this project, the educator and secondary teachers together used mainly the original texts in developing the historical materials in teaching calculus. They expanded and elaborated on them to match existing teaching materials. During the implementation of the teaching, since some of the materials were quite abundant, it was feared that it overwhelmed the students. Thus, the lesson plan could be divided into smaller teaching units to facilitate teacher's instruction. Before the lesson plans are started, the research team should first consider the learning objectives and then design strategies for learning activities and devise tests to evaluate learning outcomes. From the research process and results, it has been found that the developed history of calculus materials have a positive effect on the students, which also shows the feasibility of integrating the history of calculus material into the high school mathematics teaching.

\section{REFERENCES}

[1] C. L. Shen and Y. W. Su, "When animation and learning sheets meet: Integrating history of mathematics into elementary teaching," New Horizon Bimonthly for Teachers in Taipei, vol. 163, pp. 70-77, Dec. 2009.

[2] M. S. Lin and Y. W. Su, "Letting mathematics being interesting by history of mathematics," The Educator Monthly, vol. 509, pp. 81-83, Nov. 2009.

[3] H. N. Tsai and Y. W. Su, "Integrating history of mathematics into elementary teaching: Multiplication and division of fractions," Taiwan Journal of Mathematics Teachers, vol. 20, pp. 17-40, Dec. 2009.

[4] W. S. Horng, "Nam Byung Gil's comments on the "Tian Yuan Shu" versus "Jie Gen Fang": An HPM perspective," Chinese Journal of Science Education, vol. 8, no. 3, pp. 215-224, Sep. 2000.

[5] W. S. Horng, J. M. Ying, Y. W. Su, H. Y. Su, Q. R. Yang, and P. H. Liu When Mathematics Meets Culture, Taipei: SanMin, 2009.

[6] National Council of Teachers of Mathematics, Curriculum and Evaluation Standards for School Mathematics, Reston, VA: National Council of Teachers of Mathematics, 1989.

[7] National Council of Teachers of Mathematics, Principles and Standards for Teaching Mathematics, Reston, VA: National Council of Teachers of Mathematics, 2000.

[8] F. Fasanelli et al., "The political context," History in Mathematics Education: The ICMI Study, Dordrecht, The Netherlands: Kluwer Academic Publishers, pp. 1-38, 2000.

[9] J. D. Lange, "Mathematics for literacy," Quantitative Literacy: Why Numeracy Matters for Schools and Colleges, Princeton, NJ: National Council on Education and Disciplines, pp. 75-89, 2003.

[10] M. Niss and T. Højgaard, "Competencies and mathematical learning: Ideas and inspiration for the development of teaching and learning in denmark (IMFUFA tekst)," Roskilde, Denmark: Roskilde University, 2011.

[11] Y. W. Su, "An initial investigation into the design of materials of history of mathematics for elementary school education," Research and Development in Science Education Quarterly, vol. 62, pp. 75-96, Sep. 2011.

[12] R. M. Panasuk and L. B. Horton, "Integrating history of mathematics into curriculum: What are the chances and constraints?" International Electronic Journal of Mathematics Education, vol. 7, no. 1, pp. 3-20, June 2012

[13] E. Barbin, "Integrating history: Research perspectives," History in Mathematics Education: The ICMI Study, Dordrecht, The Netherlands: Kluwer Academic Publishers, pp. 63-90, 2000.

[14] J. Fauvel, "Using history in mathematics education," For the Learning of Mathematics, vol. 11, no. 2, pp. 3-6, June 1991.

[15] F. Furinghetti and D. Paola, "History as a crossroads of mathematical culture and educational needs in the classroom," Mathematics in School, vol. 32, no. 1, pp. 37-41, Jan. 2003.

[16] I. Gulikers and K. A. Blom, "'A historical angle', a survey of recent literature on the use and value of history in geometrical education," Educational Studies in Mathematics, vol. 47, no. 2, pp. 223-258, July 2001.

[17] C. Tzanakis and A. Arcavi, "Integrating history of mathematics in the classroom: An analytic survey," History in Mathematics Education: The ICMI Study, Dordrecht, The Netherlands: Kluwer Academic Publishers, pp. 201-240, 2000.

[18] L. Streefland, Fractions in Realistic Mathematics Education Dordrecht: Kluwer Academic Publishers, 1991.

[19] A. Dematte and F. Furinghetti, "Hisory, figures and narratives in mathematics teaching," Recent Developments on Introducing a Historical Dimension in Mathematics Education, Washington, DC: MAA, pp. 103-112, 2011.

[20] D. Pengelley, "Teaching with primary historical sources: Should it go mainstream? Can it? "Recent Developments on Introducing a Historical Dimension in Mathematics Education, Washington, DC: MAA, pp. 1-8, 2011.

[21] Y. W. Su, "Integrating ancient text into teaching," Taiwan Journal of Mathematics Teachers, vol. 9, pp. 56-67, Mar. 2007.

[22] H. Freudenthal, Mathematics As an Educational Task. Dordrecht: D, Reidel Publishing Company, 1973.

[23] K. Gravemeijer, "Developmental research as a research method," Mathematics Education As a Research Domain: A Search for Identity (An ICMI Study) New ICMI Studies Series, vol. 1, book 2, Dordrecht: Kluwer Academic Publishers, pp. 277-295, 1989. 
[24] K. D. Michalowicz, C. Daniel, G. FitzSimons, M. V. Ponza, and W. Troy, "History in support of diverse educational requirements: Opportunities for change," History in Mathematics Education: The ICMI Study, Dordrecht, The Netherlands: Kluwer Academic Publishers, pp. $171-200,2000$

[25] C. Weeks, "Stories," Mathematics in School, vol. 32, no. 1, pp. 43-46, Jan. 2003.

[26] D. Corfield, "Narrative and the rationality of mathematical practice," Circles Disturbed: The Interplay of Mathematics and Narrative, Princeton, NJ: Princeton University Press, pp. 244-280, 2012.

[27] S. Gerofsky, "A linguistic and narrative view of word problems in mathematics education," For the Learning of Mathematics, vol. 16, no. 2, pp. 36-45, June 1996.

[28] Y. Solomon and J. O'Neill, "Mathematics and narrative," Language and Education, vol. 12, no. 3, pp. 10-221, 1998.

[29] A. Doxiadis, "Euclid's poetics: An examination of the similarity between narrative and proof," Mathematics and Culture II, Berlin: Springer-Verlag, 2005, pp. 175-182.
[30] R. S. D. Thomas, "Mathematics and narrative," The Mathematical Intelligencer, vol. 24, no. 3, pp. 43-46. June 2002

[31] F. M. Lin and W. S. Horng, "A preliminary study of math fiction: Comparisons of two novels through perspectives of structuralism and narrative analysis," Chinese Journal of Science Education, vol. 17, no. 6, pp. 531-549. Dec. 2009.

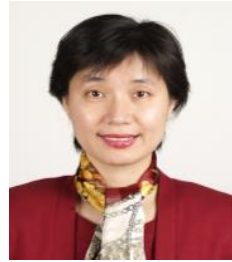

Yi-Wen Su is an associate professor in the Department of Mathematics at University of Taipei. Her research interests include integrating history of mathematics into teaching and reading comprehension in mathematics, as well as teachers' professional development. Her current research deals with the development of math reading materials and history of mathematics materials. She also devotes herself to planning and implementing teacher education courses for pre-service elementary school teachers and professional programs for in-service mathematics teachers. 